\title{
ARTICLE
}

\section{Mid-infrared ultra-high-Q resonators based on fluoride crystalline materials}

\author{
C. Lecaplain ${ }^{1, \star}$, C. Javerzac-Galy ${ }^{1, \star}$, M.L. Gorodetsky ${ }^{2,3}$ \& T.J. Kippenberg ${ }^{1}$
}

The unavailability of highly transparent materials in the mid-infrared has been the main limitation in the development of ultra-sensitive molecular sensors or cavity-based spectroscopy applications. Whispering gallery mode microresonators have attained ultra-high-quality (Q) factor resonances in the near-infrared and visible. Here we report ultra-high $Q$ factors in the mid-infrared using polished alkaline earth metal fluoride crystals. Using an uncoated chalcogenide tapered fibre as a high-ideality coupler in the mid-infrared, we study via cavity ringdown technique the losses of $\mathrm{BaF}_{2}, \mathrm{CaF}_{2}, \mathrm{MgF}$ and $\mathrm{SrF}_{2}$ microresonators. We show that $\mathrm{MgF}_{2}$ is limited by multiphonon absorption by studying the temperature dependence of the $\mathrm{Q}$ factor. In contrast, in $\mathrm{SrF}_{2}$ and $\mathrm{BaF}_{2}$ the lower multiphonon absorption leads to ultra-high $Q$ factors at $4.5 \mu \mathrm{m}$. These values correspond to an optical finesse of $\mathcal{F}>1 \times 10^{5}$, the highest value achieved for any type of mid-infrared resonator to date.

\footnotetext{
${ }^{1}$ École Polytechnique Fédérale de Lausanne (EPFL), CH-1015 Lausanne, Switzerland. ${ }^{2}$ Russian Quantum Center, 143025 Skolkovo, Russia. ${ }^{3}$ Faculty of Physics, M.V. Lomonosov Moscow State University, 119991 Moscow, Russia. ${ }^{*}$ These authors contributed equally to this work. Correspondence and requests for materials should be addressed to T.J.K. (email: tobias.kippenberg@epfl.ch).
} 
T he mid-infrared (mid-IR) spectral window $(\lambda \sim 2.5-20 \mu \mathrm{m})$ is a very useful range for spectroscopy, chemical and biological sensing, materials science and industry as it includes strong rotational-vibrational absorption lines of many molecules, as well as two atmospheric transmission windows of 3-5 and $8-13 \mu \mathrm{m}$. In this band, the absorption strengths of molecular transitions are typically 10 to 1,000 times greater than those in the visible or near-infrared (near-IR), offering the potential to identify the presence of substances with extremely high sensitivity and selectivity, as required in trace gas detection ${ }^{1-3}$, breath analysis ${ }^{4,5}$ and pharmaceutical process monitoring. The invention of the quantum cascade laser $(\mathrm{QCL})^{6}$ was a scientific and technological milestone in the development of mid-IR laser sources. Today, continuous-wave QCLs operate at room temperature, offer high output power (watts) and are a commercial technology ${ }^{7}$. These features lead to their wide adoption for spectroscopic applications in the mid-IR region. However, their free running linewidth is subject to undesired noise that makes their frequency stabilization challenging ${ }^{8}$. High finesse optical cavities have the potential for substantial reduction of laser noise $e^{9,10}$ and have been recently investigated ${ }^{11}$.

The exploration of ultra-high-quality $(Q)$ cavities in the mid-IR is a relatively new area driven by the development of QCL frequency stabilization ${ }^{11}$, ultra-sensitive molecular sensors ${ }^{12}$, cavity-based spectroscopy ${ }^{4,13}$ and optical frequency combs ${ }^{14}$. ZBLAN $^{15}$ and chalcogenide $(\mathrm{ChG})$ glass ${ }^{16}$ resonators also appear as promising for mid-IR wavelengths. Moreover, the silicon platform ${ }^{17}$ has enabled the fabrication of mid-IR microresonators at $\lambda \sim 5 \mu \mathrm{m}$. To date, $Q$ factors of $10^{5}$ are already achieved in silicon-on-sapphire ${ }^{18}$ and ChG glass-on-silicon ${ }^{19}$, whereas $Q$ factors of $10^{4}$ are obtained in silicon-on-calcium fluoride ${ }^{20}$. Yet to date, achieving ultra-high $Q$ factors in the mid-IR (or correspondingly high optical finesse) is an outstanding challenge. In the near-IR, high finesse is achieved using Fabry-Pérot cavities based on supermirrors ${ }^{21}$ or using microcavities based on whispering gallery mode (WGM). Interference coatings based on substrate-transferred singlecrystal multilayers show the potential for $\leq 100$ p.p.m. of optical losses in the mid-IR (potentially out to $7 \mu \mathrm{m})^{22}$.

Owing to high purity and low material losses, crystalline WGM microresonators ${ }^{23}$ exhibit the highest $Q$ factors in the near-IR and visible spectral ranges (along with the highest reported optical finesse of $\mathcal{F}=10^{7}$ as in ref. 24). Ultra-high $Q$ factors $\left(>10^{8}\right)$ are routinely obtained in magnesium fluoride $\left(\mathrm{MgF}_{2}\right)$ and calcium fluoride $\left(\mathrm{CaF}_{2}\right)^{25,26}$, and can be as high as $10^{11}$ in $\mathrm{CaF}_{2}$ (ref. 24). Recently, soft fluoride crystals such as barium fluoride $\left(\mathrm{BaF}_{2}\right)$ and strontium fluoride $\left(\mathrm{SrF}_{2}\right)$ have equally demonstrated ultra-high $Q$ in the near- $\operatorname{IR}^{27,28}$. Such ultra-high $Q$ factors have allowed for the generation of Kerr combs ${ }^{23}$, as well as temporal dissipative soliton formation ${ }^{29,30}$ at low $(\mathrm{mW})$ power levels, and enabled low phase noise microwave generation ${ }^{23}$, as well as ultra narrow-linewidth lasers ${ }^{10}$. However, the mid-IR is a largely unchartered territory in what concerns ultra-high- $Q$ optical microresonators and material properties have not been investigated thoroughly. There have been several recent studies investigating mid-IR properties of the important class of crystalline microresonators $11,31,32$. Using QCLs, recent work has reported $Q$ factors of $\sim 10^{7}$ at $4.5 \mu \mathrm{m}$ in $\mathrm{MgF}_{2}$ (ref. 31), $\mathrm{CaF}_{2}$ (refs $11,31,32)$ and $\mathrm{BaF}_{2}$ microresonators ${ }^{31}$, which is more than two orders of magnitude lower than the near-IR values. Unfortunately, the challenges in measuring $Q$ factors have led to some nonphysical values being reported in literature, that is, exceeding the theoretical limit imposed by multiphonon absorption at this wavelength ${ }^{32}$. Therefore, it is of utmost importance to provide an authoritative and accurate account of the mid-IR quality factors above $4 \mu \mathrm{m}$-the range where
QCLs operate to guide future experiments in this wavelength range.

Here we study systematically the optical $Q$ factors of four crystalline materials transparent in the mid-IR spectrum from the alkaline earth metal fluoride $X \mathrm{~F}_{2}$ family (where $X=\mathrm{Ca}, \mathrm{Mg}, \mathrm{Ba}$ and Sr). Note that other combinations such as $X=B e$ and Ra are water soluble or toxic and therefore not suitable. We directly compare the $Q$ factor at $1,555,2,000,3,000$ and $4,500 \mathrm{~nm}$ and, importantly, observe that the intrinsic material absorption in $\mathrm{MgF}_{2}$ poses a limit to the $Q$ factor, which is greatly reduced in $\mathrm{BaF}_{2}$ and $\mathrm{SrF}_{2}$. This demonstrates that material absorption limits the $Q$ factor in this class of microresonators, because in the visible and near-IR spectral ranges the intrinsic absorption is many orders of magnitude lower.

To measure accurately the losses of crystalline materials in the mid-IR, we developed an efficient coupling technique based on an optical tapered fibre made out of ChG glass. It allows for light from a QCL to be evanescently coupled to a crystalline microresonator via a ChG-uncoated tapered fibre. We show that critical coupling $^{33}$ is achieved with high ideality ${ }^{34}$, necessary for faithful $Q$ factor measurements, and extend this technique to the mid-IR. We measured a critically coupled factor of $Q_{c} \sim 1 \times 10^{7}$ for a $\mathrm{MgF}_{2}$ microresonator, a value at the theoretical limit of multiphonon absorption at this wavelength ${ }^{31}$. To further verify the intrinsic multiphonon behaviour of $\mathrm{MgF}_{2}$, we undertook temperature-dependence measurements. Theory predicts that $\mathrm{MgF}_{2}$ microresonators that are material loss limited, should exhibit a temperature dependent $Q$ factor ${ }^{35}$, and the data presented here demonstrate the temperature variation of the multiphonon losses. This variation is consistently not observed with $\mathrm{SrF}_{2}$ or $\mathrm{BaF}_{2}$, which are not limited by this process. Using a cavity ringdown method, we demonstrate ultra-high- $Q$ mid-IR resonances. This is only possible with the proper choice of crystals, which opposite to previous work ${ }^{32}$, implies not using $\mathrm{MgF}_{2}$ but rather $\mathrm{BaF}_{2}$ or $\mathrm{SrF}_{2}$. The measured mid-IR resonances feature ultra-high intrinsic factors of $Q_{0} \geq 6 \times 10^{8}$ at $4.4 \mu \mathrm{m}$ (more than a 10 -fold improvement compared with previous results), exhibiting the highest observed finesse of $\mathcal{F} \sim 1.5 \times 10^{5}$ for any cavity in the mid-IR so far. These losses, when expressed as mirror losses in an equivalent Fabry-Pérot cavity, correspond to a mirror loss of $\sim 50$ p.p.m. at $4.4 \mu \mathrm{m}$.

\section{Results}

Mid-IR crystalline microresonators properties and fabrication. We are interested in crystalline materials whose transparency window includes the region of 3-5 $\mu \mathrm{m}$, a range, where QCL are commercially available. We studied four different crystalline materials such as $\mathrm{MgF}_{2}, \mathrm{CaF}_{2}, \mathrm{BaF}_{2}$ and $\mathrm{SrF}_{2}$, which are available commercially with high purity (and used, for example, for deep ultraviolet lenses). These crystals feature ultra-high $Q$ in the near-IR ${ }^{25-28}$ and anomalous group velocity dispersion in the mid-IR, a requirement for Kerr comb and (bright) temporal soliton formation based on the Kerr nonlinearity. However, the theoretically predicted limiting loss mechanism of the $Q$ factor in the mid-IR results from multiphonon absorption processes ${ }^{36-38}$ involving the coupling of the incident light with fundamental molecular vibrational modes of the material, a loss mechanism not observed so far in the near-IR in such crystals. Usually this reduces the mid-IR cutoff wavelength to a much shorter value than that of the transparency window's maximum wavelength. In the multiphonon-dominated regime the absorption coefficient is given by $\alpha=A e^{-\beta / \lambda}$ and largely surpasses any Rayleigh scattering contribution that is proportional to $\frac{B}{\lambda^{4}}$, where $A, \beta$ and $B$ are materials properties ${ }^{38}$. Therefore, the $Q$ factor limit is given by $\frac{2 \pi n}{\alpha \lambda}$, where $n$ is the refractive index and $\lambda$ the wavelength. 

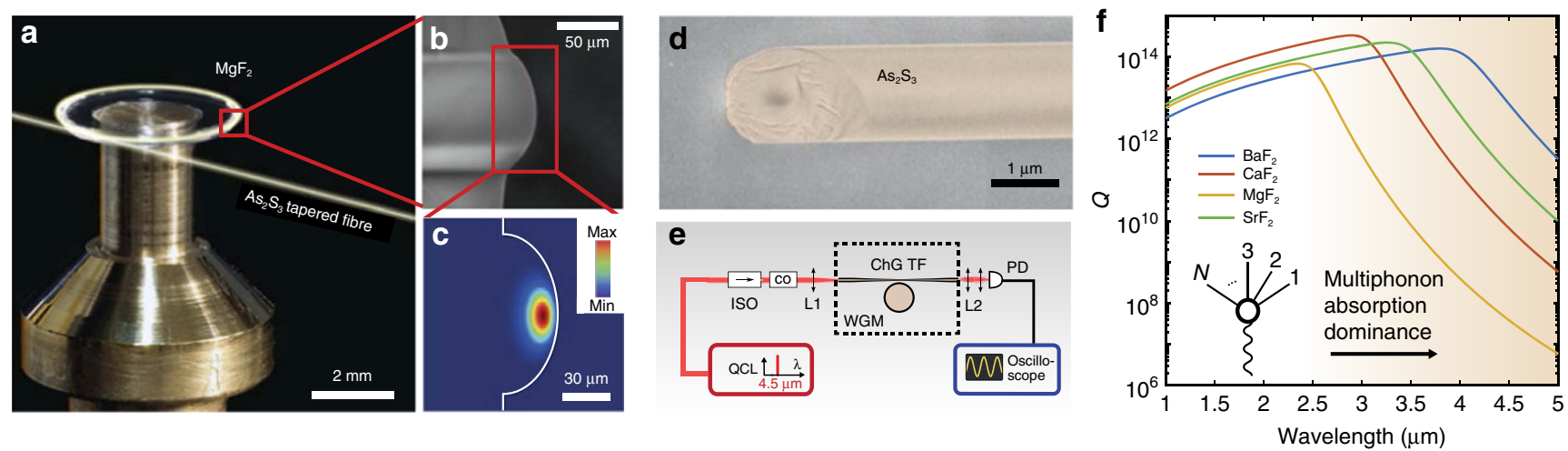

Figure 1 | Mid-IR crystalline microresonators and uncoated ChG tapered fibre. (a) $\mathrm{MgF}_{2}$ crystalline microresonator with a diameter of $\sim 5 \mathrm{~mm}$. WGMs of the microresonator are excited via evanescent coupling using a $\mathrm{ChG}$, that is, $\mathrm{ChG}\left(\mathrm{As}_{2} \mathrm{~S}_{3}\right.$ ) tapered fibre. (b) Scanning electron microscope (SEM) image of the $\mathrm{MgF}_{2}$ protrusion. Its radius of curvature, which confines the mode in the azimuthal direction, is $\sim 50 \mu \mathrm{m}$. (c) Finite element model simulations of the optical intensity profile of the fundamental WGM at $\lambda=4.5 \mu \mathrm{m}$. (d) SEM image of the waist of a ChG tapered fibre with subwavelength diameter of $1.2 \mu \mathrm{m}$. (e) Experimental set-up composed of a WGM microresonator pumped by a QCL evanescently coupled through a ChG tapered fibre (ChG TF), followed by an oscilloscope to record the transmission. An optical isolator (ISO) protects the pump laser from Fresnel reflection ( $\sim 14 \%)$ at the cleaved fibre ends. Mid-IR free space control optics (CO), including waveplates, neutral densities and a mid-IR electro-optic modulator. L1 and L2 are lenses for free space coupling into the ChG TF. PD, photodetector. Tapered fibre and microresonator are kept under a dry and inert atmosphere to preserve from degradation and physical ageing of ChG fibre ${ }^{43}$. (f) Quality factor dependence of different fluoride crystals with respect to the wavelength. For mid-IR wavelengths, multiphonon absorption competes with Rayleigh scattering and strongly impacts the $Q$ factor. The orange shading highlights increasing multiphonon absorption contribution. The inset depicts a $\mathrm{N}$-phonon creation process within the multiphonon absorption of one photon.

Figure If presents the dependence of the $Q$ factor on the wavelength for alkaline earth metal fluoride crystalline materials $^{39}$. It reveals that even though a material displays low Rayleigh scattering and ultra-high $Q$ in the near-IR, it will be subject to multiphonon absorption in the mid-IR that increases by $\sim 2-6$ orders of magnitude at $4.5 \mu \mathrm{m}$ compared with $1.5 \mu \mathrm{m}$ for these crystal families. On the basis of theoretical considerations alone, $\mathrm{BaF}_{2}$ and $\mathrm{SrF}_{2}$ seem ideal candidates to achieve ultra-high $Q$ in the mid-IR in comparison with $\mathrm{MgF}_{2}$ due to lower multiphonon absorption. Though there has been considerable interest in highly transparent infrared materials for the last decades, only spectrophotometric measurements were carried out to study absorption due to technical limitations of using microresonators in the mid-IR ${ }^{36-38}$. We emphasize that conventional methods (for example, Fourier transform infrared spectroscopy) to investigate loss are not suitable to probe the losses at the level of p.p.m., as required to achieve ultra-high $Q$.

We fabricated our microresonators either from disk or cylinder blanks. The microresonators were first shaped by grinding or diamond-cutting tool and polished in an air-bearing spindle by successive smaller diamond particle slurries to obtain a smooth protrusion as visualized in Fig. 1b. Their final diameters are $\sim 5 \mathrm{~mm}$. We analysed the microresonators in a scanning electron microscope, and from this we determined the transverse radii of the crystalline $\mathrm{MgF}_{2}$ disk to be of $\lesssim 50 \mu \mathrm{m}$. The intensity profile of the fundamental WGM of the $\mathrm{MgF}_{2}$ microresonator in the mid-IR is displayed in Fig. 1c. From finite element model simulations we obtained an effective mode area of $A_{\text {eff }} \sim 600 \mu \mathrm{m}^{2}$ at $\lambda \sim 4.4 \mu \mathrm{m}$. To ensure that we are not limited by scattering losses from residual surface roughness, optical quality factors were first measured at $\lambda=1.55 \mu \mathrm{m}$, with a tunable, narrowlinewidth (short-term $<100 \mathrm{kHz}$ ) fibre laser using silica tapered fibres to excite the $\mathrm{WGM}^{26}$. The $\mathrm{MgF}_{2}$ disk features loaded optical factors of $Q \geq 5 \times 10^{8} ; \mathrm{CaF}_{2}$ and $\mathrm{SrF}_{2}$ exhibit $Q$ factors of $\sim 2 \times 10^{9}$ and the $\mathrm{BaF}_{2}$ cylinder features $Q \sim 6.4 \times 10^{9}$ (detailed below and in Fig. 5).

ChG tapered fibres as high-ideality mid-IR couplers. To measure far in the infrared, we used a mode-hop-free tunable QCL
(Daylight Solutions Inc.). To couple QCL light into the microresonator we developed uncoated optical tapered fibres made out of ChG glass ${ }^{40,41}$. ChG fibres are particularly attractive due to their low loss in the mid- $\mathrm{IR}^{42}$ and commercial availability. However, they were never used previously as a low-loss, uncoated tapered optical waveguide because of technical fabrication challenges in their tapering process and maintenance, due to the low melting point of $\mathrm{ChG}$ glasses $\left(\sim 200^{\circ} \mathrm{C}\right.$ for $\left.\mathrm{As}_{2} \mathrm{~S}_{3}\right)$. We have successfully developed a tapering set-up with a feedback controlled electrical heater to adiabatically pull low-loss uncoated tapered ChG fibres. To verify the theoretically expected taper waist, scanning electron microscope images were taken to optimize the tapering process. The ChG $\left(\mathrm{As}_{2} \mathrm{~S}_{3}\right)$ tapered fibre was fabricated from a commercially available, IRflex IRF-S 9 nonlinear mid-IR fibre, made from extra-high-purity ChG glass (see Methods section). The fibre is transparent from 1.5 to $6.5 \mu \mathrm{m}$ and has a core diameter of $9 \mu \mathrm{m}$ and a high nonlinear refractive index of $n_{2}=2.7$. The ChG tapered fibre was pulled down to a diameter to phase match the fundamental WGM. For instance, for a $\mathrm{MgF}_{2}$ microresonator radius of $R \sim 2.5 \mathrm{~mm}$, the optimum taper waist in the mid-IR corresponds to a subwavelength diameter around $1 \mu \mathrm{m}$ (Fig. 1d). The experimental set-up is described in Fig. 1e. The pump laser is a $200 \mathrm{~mW}$ continuous wave external cavity QCL, tunable from 4.385 to $4.58 \mu \mathrm{m}$. The tapered fibre and microresonator are kept under a dry and inert atmosphere to preserve them from degradation and physical ageing of the ChG fibre ${ }^{43}$. The QCL light is evanescently coupled to the crystalline microresonator using the $\mathrm{ChG}$ tapered fibre. When the taper-resonator coupling is achieved, several resonance families are observed at $\lambda \sim 4.4 \mu \mathrm{m}$, as illustrated in Fig. 2a.

A unique property of taper-coupled microresonators is that phase matching (and thus the coupling) can be finely tuned by translating the microresonator along the taper relative to the waist ${ }^{33}$. Measuring ultra-low losses in microresonators requires an ideal coupler. We investigated in detail the mid-IR coupling behaviour between the $\mathrm{ChG}$ taper and the $\mathrm{MgF}_{2}$ crystalline microresonator for a typical mode family. For an unity ideality coupler ${ }^{34}$, the coupling parameter is given by $K=\kappa_{\mathrm{ex}} / \kappa_{0}=$ $(1 \pm \sqrt{T}) /(1 \mp \sqrt{T})$, where $\kappa_{0}$ is the intrinsic loss rate, $\kappa_{\text {ex }}$ the 
a

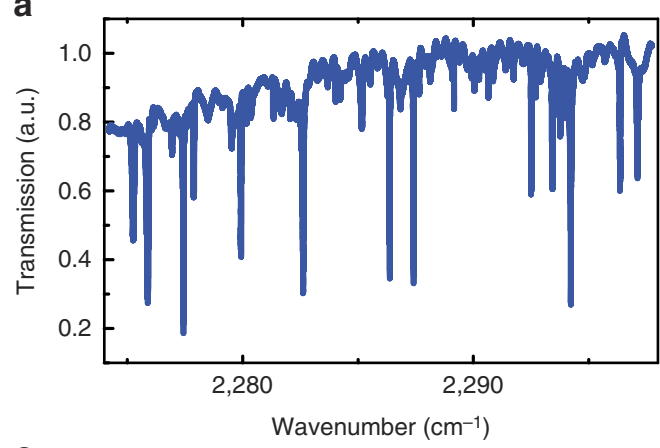

b

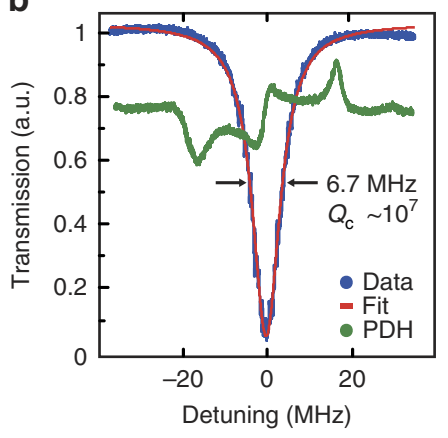

。

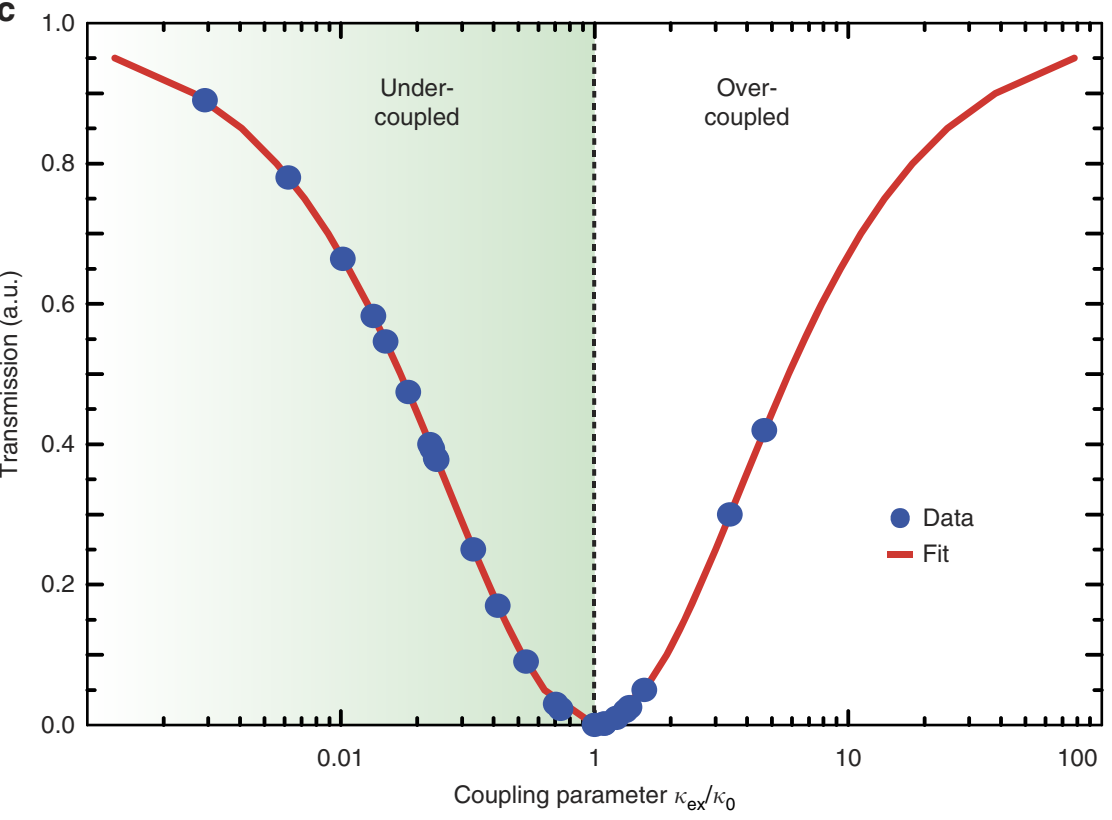

Figure 2 | Chalcogenide taper coupling efficiency around $\mathbf{4 . 5} \boldsymbol{\mu m}$ (a) Representative transmission spectrum composed of several resonance families over a wide mid-IR range when taper-resonator coupling is achieved with the $\mathrm{MgF}_{2}$ microresonator and set-up described in Fig. 1. (b) Measurement (blue curve) of a resonance linewidth at critical coupling with frequency calibration provided by a Pound-Drever-Hall (PDH) signal (green curve) and Lorentzian fit (red curve). The typical full-width at half maximum width of $\kappa / 2 \pi \sim 6.7 \mathrm{MHz}$ corresponds to a critically coupled quality factor of $Q_{\mathrm{c}} \sim 1.0 \times 10^{7}$.

(c) Transmission as a function of the coupling parameter $K=\kappa_{\mathrm{ex}} / \kappa_{0}$ for varying taper waist radius. The dashed line marks the critical coupling point $\left(\kappa_{\mathrm{ex}}=\kappa_{0}\right)$. The experimental data (blue circles) are consistent with the theoretical model $\kappa_{\mathrm{ex}} / \kappa_{0}=(1 \pm \sqrt{T}) /(1 \mp \sqrt{T})$ (red curve) demonstrating that the ChG taper behaves as a nearly ideal coupler in mid-IR with close to unity ideality.

photon loss rate due to coupling to the microresonator and $T$ the transmission on resonance. The upper signs are used for transmission values $T$ in the over-coupled regime $\left(\kappa_{0}<\kappa_{\mathrm{ex}}\right)$ and the lower signs for the under-coupled regime $\left(\kappa_{0}>\kappa_{\mathrm{ex}}\right)$. We recorded the transmission spectrum while scanning the laser over resonance for different taper waist radii. We measured the corresponding full-width at half maximum linewidth by setting up a Pound-Drever-Hall technique with a mid-IR electro-optic phase modulator (Qubig $\mathrm{GmbH}$ ) to provide a frequency calibration. Figure $2 \mathrm{c}$ shows the normalized transmission as a function of the coupling parameter $K$. Critical $\left(\kappa_{0}=\kappa_{\mathrm{ex}}\right)$ and strong overcoupling up to $K \sim 6$ are observed for optimum taper diameters. We emphasize that this represents the achievement of critical coupling in crystalline microresonators in the mid-IR region. This reveals that the ChG taper behaves as a nearly ideal coupler, with close to unity ideality ${ }^{34}$. We measured a linewidth of $\kappa / 2 \pi \sim 6.7 \mathrm{MHz}$ at critical coupling (red curve in Fig. 2b.) yielding an optical factor of $Q_{c} \sim 1.01 \times 10^{7}$ for $\mathrm{MgF}_{2}$. Our measurements reveal that uncoated $\mathrm{ChG}$ tapers can thus extend the efficient tapered fibre coupling method deep to the mid-IR regime. It enables an accurate measurement of the material losses in the mid-IR, in contrast to other methods such as prism coupling, which does not allow an ideal coupling ${ }^{31,32}$. We point out that it makes very clear that the values are indeed limited by intrinsic multiphonon absorption and not by coupling loss due to lack of ideality. As a consequence, the excellent ability to trace out the coupling curve with high ideality leaves little doubt about the fact that the internal $Q$ factor is on the order of $10^{7}$ and not higher ${ }^{32}$.

Near- and mid-IR cavity ringdown measurements. After $\mathrm{MgF}_{2}$ characterization, we studied other fluoride crystals $\left(\mathrm{CaF}_{2}, \mathrm{BaF}_{2}\right.$ and $\mathrm{SrF}_{2}$ ) and first characterized them in the near-IR set-up. We measured the optical $Q$ factor using both frequency modulation spectroscopy and a swept laser cavity ringdown technique. The cavity ringdown method enables a measurement of the quality factor independently of the thermal nonlinearity (hence at higher pumping powers) and of the laser linewidth. Figure 3a shows a typical resonance obtained in the under-coupled regime for a pump power of $P \leq 1 \mathrm{~mW}$ in $\mathrm{BaF}_{2}$. The Lorentzian fit gives a full-width at half maximum linewidth of $\kappa / 2 \pi \sim 44 \mathrm{kHz}$ at 
a

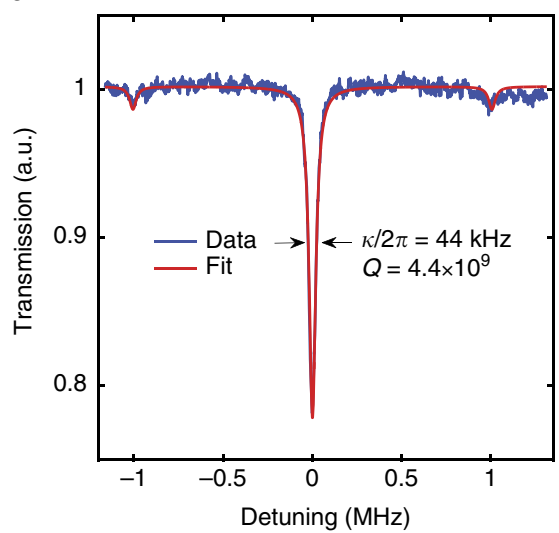

b

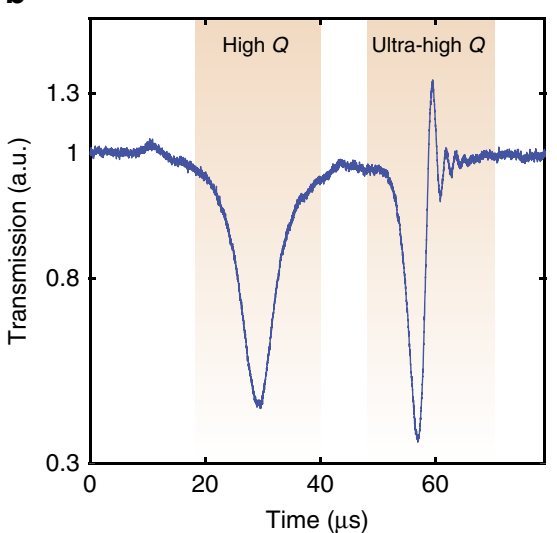

C

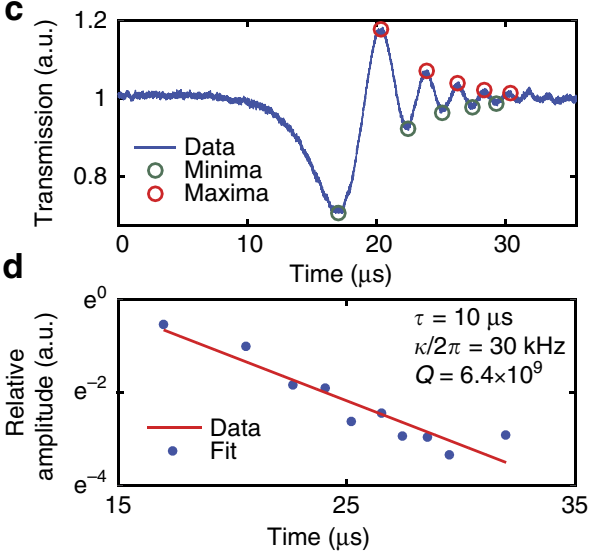

Figure $\mathbf{3}$ | Near-IR characterization of $\mathbf{a} \mathbf{B a F}_{\mathbf{2}}$ microresonator. (a) Linewidth measurement (blue line) of an under-coupled resonance with calibration sidebands (resulting from phase modulation of the laser) at $1 \mathrm{MHz}$ and Lorentzian fit (red line). We extract a typical full-width at half maximum of $\kappa / 2 \pi \sim 44 \mathrm{kHz}$ resulting in an optical factor of $Q \sim 4.4 \times 10^{9}$. (b) Cavity-ringdown measurements. We observed the transmission spectrum of two resonances while scanning at the same speed. Only the ultra-high-Q mode features a ringdown signal (right resonance). (c) Analysis of ringdown structure. We extract successive amplitudes of maxima (red circles) and of minima (green circles) to fit the evolution of the relative amplitude ringdown (d). Theoretical fit of the ringdown relative amplitude (red line). The measured amplitude decay of $\tau=10 \mu \mathrm{s}$ results in a linewidth of $\kappa / 2 \pi=30 \mathrm{kHz}$ and a $Q$ factor of $6.4 \times 10^{9}$.
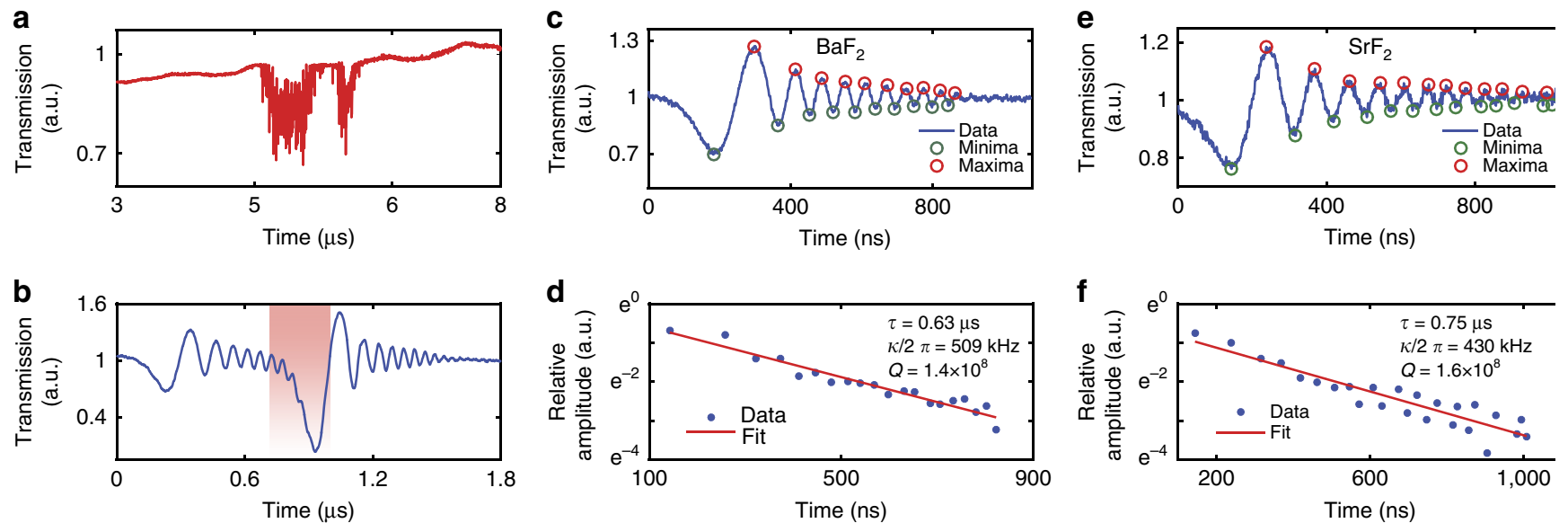

Figure 4 | Mid-IR swept frequency ringdowns of $\mathbf{B a F}_{\mathbf{2}}$ and $\mathbf{S r F}_{\mathbf{2}}$ microresonators. (a) Thermal distortions of a resonance in the $\mathrm{BaF}_{2} \mathrm{Crystalline}$ microresonator. Thermal-optical dynamics prevent measuring the linewidth and the transmission accurately using standard frequency modulation spectroscopy method. (b) Using a cavity swept laser ringdown method, we observe modal coupling between two ultra-high- $Q$ resonances (red shading). $(\mathbf{c}, \mathbf{e})$ Transmission spectra of the ringdown signal of an ultra-high-Q resonance in mid-IR at $4.4 \mu \mathrm{m}$ using $\mathrm{BaF}_{2}(\mathbf{c})$ and $\mathrm{SrF}_{2}(\mathbf{e})$. (d,f) Exponential fits (red lines) of the minima and maxima data sets (blue circles) extracted from $\mathbf{c}, \mathbf{e} . \ln \mathrm{BaF}_{2}$ (d), the measured amplitude lifetime of $\tau=0.63 \mu$ s results in a linewidth of $\kappa / 2 \pi=509 \mathrm{kHz}$ and a corresponding $Q$ factor of $\sim 1.4 \times 10^{8}$. In $\mathrm{SrF}_{2}(\mathbf{f})$, the measured amplitude lifetime of $\tau=0.75 \mu$ s results in a linewidth of $\kappa / 2 \pi=430 \mathrm{kHz}$ and a corresponding $Q$ factor of $\sim 1.6 \times 10^{8}$.

$1,555 \mathrm{~nm}$, resulting in an optical factor of $Q \sim 4.4 \times 10^{9}$. From the coupling curve we can extract moreover an intrinsic linewidth of $\kappa_{0} / 2 \pi \sim 22 \mathrm{kHz}$ (corresponding to an intrinsic $Q$ factor $Q_{0}$ of $\left.0.9 \times 10^{10}\right)$. In addition, we performed swept laser cavity ringdown measurements ${ }^{26}$ to first calibrate the method in the near-IR. When an ultra-high- $Q$ resonator is excited with a laser whose frequency is linearly swept across the resonance with a duration shorter than the cavity lifetime, its transmission spectrum shows oscillations (Fig. 3b). These oscillations result from the beating of the transiently build-up light inside the resonator that decays into the fibre, with that of the swept laser source. Importantly, when the laser is swept fast, the two components will have a different beat frequency giving rise to an exponentially decaying oscillation. In the case of a lower $Q$ mode (with negligible cavity build-up), no ringdown signal is observed at the same scan speed, as highlighted in Fig. 3b. We analysed the transmission spectrum of the ringdown structure in Fig. $3 \mathrm{c}$ by measuring the amplitude decay $\tau$ of the remitted light ${ }^{24}$. Its theoretical fit (Fig. 3d) gives a measured amplitude decay $\tau$ of $10 \mu \mathrm{s}$, corresponding to an intrinsic cavity factor $Q_{0}=Q \times(1+K)=\omega \tau / 2 \times(1+K) \sim 2.8 \times 10^{10}\left(Q \sim 6.4 \times 10^{9}\right)$. This value is close to the one derived with the frequency modulation spectroscopy, corroborating the method. $\mathrm{In}_{\mathrm{CaF}}$ and $\mathrm{SrF}_{2}$ microresonators we measured $\tau \sim 3 \mu \mathrm{s}\left(Q \sim 1.8 \times 10^{9}\right)$, corresponding to an intrinsic cavity factor $Q_{0} \sim 6.8 \times 10^{9}$, in agreement with the values derived from frequency modulation spectroscopy. We emphasize that we did not resort to annealing procedures to improve $Q$ factors of our microresonators ${ }^{24}$.

Having established the ultra-high- $Q$ resonance in the near-IR, and having developed the mid-IR ChG tapered fibre method, we afterwards measured $Q$ factors in the mid-IR. Phase matching between the ChG tapered fibre and the microresonator is 

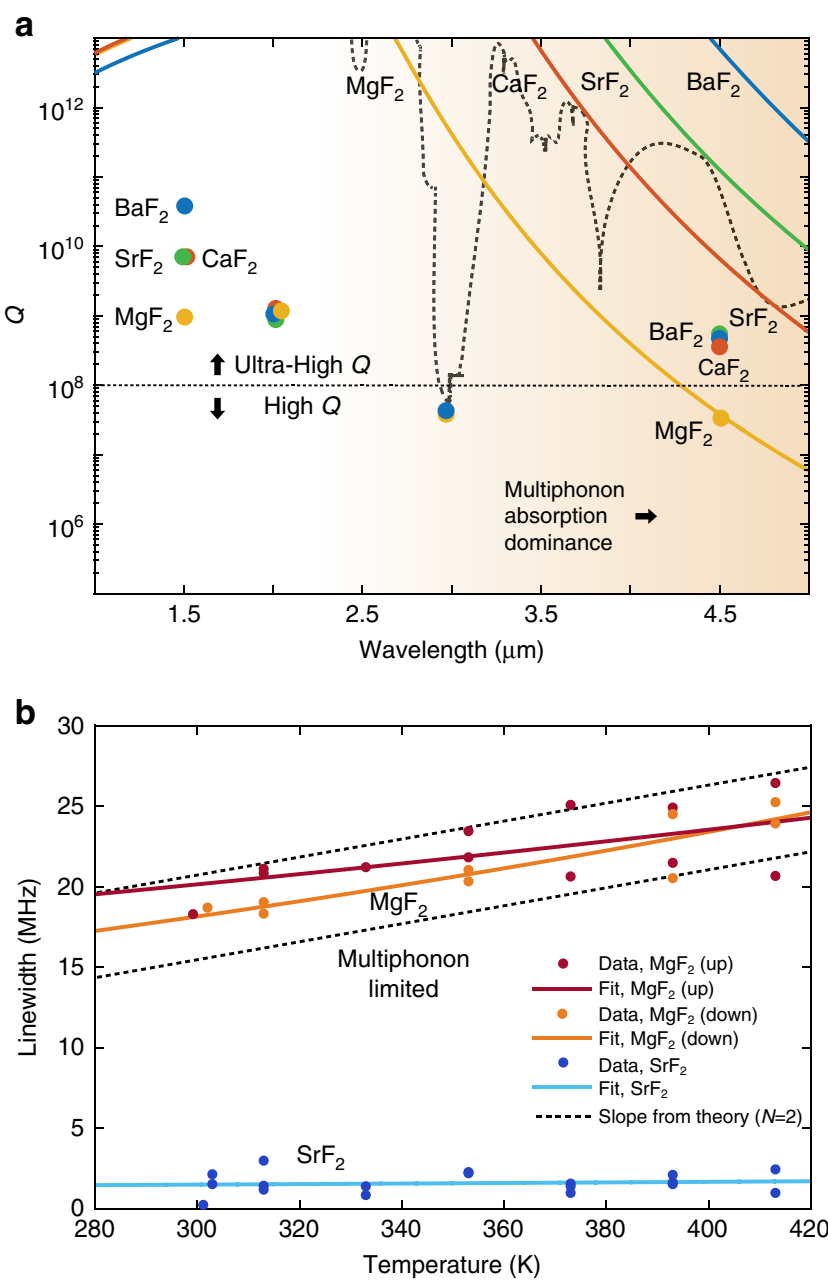

Figure 5 | Mid-IR multiphonon absorption in crystalline microcavities.

(a) Measurements for different fluoride crystals of the $X F_{2}$ family (where $X=\mathrm{Ca}, \mathrm{Mg}, \mathrm{Ba}$ and $\mathrm{Sr}$ ) prove the possibility of attaining the ultra-high-Q regime in the mid-IR. Except for $\mathrm{MgF}_{2}$, for which we reach the theoretical limit imposed by multiphonon absorption (orange shaded region) at room temperature, other materials offer $Q \geq 10^{8}$ around $4.5 \mu \mathrm{m}$. The lines represent the theoretical multiphonon absorption limit of $Q$ with respect to the wavelength. The circles represent our experimental values. Despite the clear differences with the near-IR region, mid-IR cavities are able to overcome the high- $Q$ regime achieving $Q>10^{8}$. Measurements around $2 \mu \mathrm{m}$ show typical level of impurities and defects that limits quality factors when they are not limited by Rayleigh scattering (short wavelengths) or multiphonon absorption (long wavelengths). Measurements around $3 \mu \mathrm{m}$ highlight that $\mathrm{OH}$ absorption can strongly degrade the intrinsic $Q$ factor of crystalline materials and constitutes a lower bound of $Q$ limitation due to impurities and defects. The dashed grey line is a guide to the eye depicting bulk water absorption $Q$ limitation (from ref. 50 ). Note that $1.5 \mu \mathrm{m}$ represents the less-affected wavelength by $\mathrm{OH}$ absorption (by a few orders of magnitude compared with any other wavelengths). (b) Experimental proof of multiphonon absorption in a microresonator made out of an ionic crystal. Temperature sweeps of the microresonator reveal a typical temperature dependence of intrinsic multiphonon absorption for $\mathrm{MgF}_{2}$ (fitted slopes of $\sim 4 \times 10^{-2} \mathrm{MHzK}^{-1}$ ) and consistently no temperature dependence for extrinsic absorption limited $\mathrm{SrF}_{2}$ (fitted slope of $10^{-3} \mathrm{MHzK}^{-1}$ ). Sweep with increasing (up) and decreasing (down) temperatures corroborate the temperature dependence of the intrinsic losses. From the theoretical slope (see Discussion) we infer that twophonon $(N=2)$ processes contribute mostly to the temperature dependence.
Table 1 | Measured properties of different crystalline fluoride materials at $4.4 \mu \mathrm{m}$.

\begin{tabular}{lccccc} 
Crystal & $\boldsymbol{\kappa} / \mathbf{2} \boldsymbol{\pi}$ & $\mathbf{Q}$ & $\mathbf{Q}_{\mathbf{0}}$ & $\boldsymbol{\alpha} \mathbf{( c m}^{-\mathbf{1}} \mathbf{)}$ & $\mathcal{F}$ \\
\hline $\mathrm{MgF}_{2}$ & $3.4 \mathrm{MHz}$ & $1 \times 10^{7}$ & $2 \times 10^{7}$ & $9 \times 10^{-4}$ & $4 \times 10^{3}$ \\
$\mathrm{CaF}_{2}$ & $710 \mathrm{kHz}$ & $9.6 \times 10^{7}$ & $3.5 \times 10^{8}$ & $5 \times 10^{-5}$ & $7.8 \times 10^{4}$ \\
$\mathrm{BaF}_{2}$ & $509 \mathrm{kHz}$ & $1.4 \times 10^{8}$ & $6.2 \times 10^{8}$ & $3.3 \times 10^{-5}$ & $1.3 \times 10^{5}$ \\
$\mathrm{SrF}_{2}$ & $430 \mathrm{kHz}$ & $1.6 \times 10^{8}$ & $6 \times 10^{8}$ & $3.2 \times 10^{-5}$ & $1.5 \times 10^{5}$ \\
\hline
\end{tabular}

achieved by translating the taper position. In contrast to the nearIR, when coupling is achieved, we observed large thermal instability in the mid- $\mathrm{IR}^{44}$. These thermal instabilities induce large distortions of the resonance (Fig. $4 \mathrm{a}$ ) preventing any reliable measurement of its linewidth ${ }^{24,31}$ using the Pound-Drever-Hall error signal as a calibration. In that case, the optical $Q$ factor can only be obtained by cavity ringdown measurements. We scanned the QCL using its laser current modulation to observe the cavity ringdown signal. Amplitude and cavity lifetime depend on coupling and linewidth of the resonance. When two resonances are really close, we observe a modal coupling between the two oscillatory decaying signals (Fig. $4 \mathrm{~b})^{45}$. The $\mathrm{BaF}_{2}$ transmission spectrum of a typical ringdown structure is displayed in Fig. 4c. By measuring the amplitude decay $\tau$ of the remitted light (Fig. 4d), we obtained a $\mathrm{BaF}_{2}$ intrinsic $Q$ factor of $Q_{0} \sim 6.2 \times 10^{8}$. $\mathrm{CaF}_{2}$ and $\mathrm{SrF}_{2}$ crystals were studied by applying the same methods. The ringdown analysis in the case of $\mathrm{SrF}_{2}$ is presented in Fig. 4e. We measured an amplitude decay time $\tau$ of $0.75 \mu \mathrm{s}$ (Fig. 4f), resulting in an intrinsic $Q$ factor of $Q_{0} \sim 6 \times 10^{8}$. We note that no ringdown was observed when measuring the $Q$ factor of the $\mathrm{MgF}_{2}$ microresonator under the same conditions, confirming that $\mathrm{MgF}_{2}$ does not feature ultra-high $Q$ factors in the mid-IR.

\section{Discussion}

The absorption of infrared radiation in the transparency window (absorption level of $10^{-3} \mathrm{~cm}^{-1}$ or below) can either be attributed to intrinsic processes involving several phonons or to extrinsic effects involving impurities, defects and surfaces. The difference between intrinsic and extrinsic absorption can be determined by studying the temperature dependence of the absorption. In particular, theory predicts that an exponential temperature dependence is typical of intrinsic multiphonon absorption $^{35}$. The theory considers phonons as bosons, which obey Bose-Einstein occupation of the lattice vibration modes, and thus leads to a dependence of the absorption coefficient in temperature $\alpha \sim\left(\frac{k T}{\hbar \tilde{\omega}}\right)^{N-1}$ (where the phonon frequency $\tilde{\omega}$ in a $N$-phonon process follows $\left.N \tilde{\omega}=\omega_{\text {photon }}\right)$. To further verify the intrinsic multiphonon behaviour, we thus measured the quality factor versus temperature by heating the microresonator from room temperature to $420 \mathrm{~K}$ for two crystalline materials, for example, $\mathrm{MgF}_{2}$ and $\mathrm{SrF}_{2}$. The melting of the $\mathrm{ChG}$ fibre around $470 \mathrm{~K}$ does not allow the temperature to be increased further. For $\mathrm{SrF}_{2}$, ringdown measurements show that the $Q$ factor value is independent of the temperature, whereas a temperature dependence of the $Q$ factor is observed in $\mathrm{MgF}_{2}$ (Fig. 5b), confirming that $\mathrm{MgF}_{2}$ is limited by multiphonon absorption at $4.5 \mu \mathrm{m}$ and $\mathrm{SrF}_{2}$ is not. From the theoretical slope we infer that two-phonon $(N=2)$ processes contribute mostly to the temperature dependence. Extrinsic effects such as impurities can be attributed to the $Q$ factor limit of the other crystals. For this purpose, we investigated the $Q$ factor at 2 and at $3 \mu \mathrm{m}$ using a thulium-doped fibre amplifier and an optical parametric oscillator, respectively. Using uncoated $\mathrm{ChG}$ tapered fibre and ringdown measurements, we measured similar ultra-high- $Q$ 
factors for all the crystalline materials at $2 \mu \mathrm{m}$, corresponding to intrinsic $Q$ factors of $Q_{0} \approx 10^{9}$. Assigning the origins of the residual extrinsic absorption are delicate due to experimental difficulties and uncertainty of the origins of the absorption themselves. However, we have demonstrated that intrinsic multiphonon absorption limits the $Q$ factor of $\mathrm{MgF}_{2}$ at $4.5 \mu \mathrm{m}$. At very low absorption levels of $10^{-4}$, surface absorption can also be important. Interestingly, typical internal reflection spectroscopy spectra show strong $\mathrm{OH}$ (water) absorption near $3 \mu \mathrm{m}$. Our measurements at $3 \mu \mathrm{m}$ highlight that $\mathrm{OH}$ absorption can strongly degrade the $Q$ factor of crystalline materials and constitutes a lower bound of the usually flat $Q$ limitation due to impurities and defects. The performance of all crystalline materials reveals intrinsic factors of $Q_{0} \approx 10^{7}$ near $3 \mu \mathrm{m}$. Note that we checked that the decrease of the $Q$ factors was not caused by a degradation of the microresonators. For this purpose, they were re-measured at $1,555 \mathrm{~nm}$ directly after these new measurements, showing that their ultra-high $Q$ factors did not change. Table 1 and Fig. 5 summarize our experimental results. From these measurements we extracted the limiting values of the mid-IR intrinsic absorption $\alpha \leq \frac{2 \pi n}{Q_{0} \lambda}$ at $4.4 \mu \mathrm{m}$. We show that the absorption can thus be as low as 50 p.p.m. per cm for $\mathrm{SrF}_{2}$. Translated to a single round trip in a Fabry-Pérot cavity of length $L / 2$ (where $L=2 \pi r$ for a WGM of radius $r$ ) and finesse $\mathcal{F}$, this value amounts to optical losses (absorbance) of the order of $\alpha L=\frac{2 \pi}{\mathcal{F}}$, that is, 50 p.p.m.

In summary, we have demonstrated mid-IR ultra-high- $Q$ crystalline resonators made from commercially available $\mathrm{BaF}_{2}$ and $\mathrm{SrF}_{2}$ crystals at $4.5 \mu \mathrm{m}$, a region of high interest due to the transparency of the atmosphere and absorption of toxic or greenhouse molecules (for example, $\mathrm{CO}, \mathrm{CO}_{2}, \mathrm{SO}_{2}$ or $\mathrm{CO}_{3}$ ) and the availability of QCL sources. Our results in the mid-IR verify the expected temperature variation of the multiphonon losses. Consequently, we show that the intrinsic material absorption in $\mathrm{MgF}_{2}$ poses a limit to the $Q$ factor, which is overcome in $\mathrm{BaF}_{2}$ and $\mathrm{SrF}_{2}$. We demonstrate that uncoated ChG tapered fibres can be ideal and efficient couplers deep to the mid-IR. Together with cavity ringdown methods, our platform enables precise measurements of quality factor, overcoming previous limitations. We show that a finesse as high as $\mathcal{F} \sim 1.5 \times 10^{5}$ is achievable with $\mathrm{BaF}_{2}$ and $\mathrm{SrF}_{2}$ in the mid-IR for cavities as small as few millimetres in diameter. This finesse represents more than one order of magnitude improvement over prior high finesse cavities in this wavelength range $e^{3,46,47}$. The exceptional $Q$ factor achieved in the mid-IR paves the way for the next generation of ultranarrow sources and ultra-stable cavities in the molecular fingerprint region and can further leverage QCL technology, by, for example, enabling injection-locked QCL similar to technology developed in the near-IR ${ }^{10,30}$. Despite the differences with nearIR, we prove that the mid-IR region is not limited to the high- $Q$ regime when proper materials are used. The materials' crystalline nature leads to low thermodynamical noise ${ }^{21}$ and moderate multiphonon absorption in the highly relevant mid-IR region. In addition, combining QCL with mid-IR ultra-high- $Q$ crystalline microresonators opens a route for mid-IR Kerr comb or soliton generation ${ }^{29,48,49}$.

\section{Methods}

The fabrication of uncoated ChG tapered fibres. To enable repeatable fabrication of high-quality uncoated $\mathrm{ChG}$ taper, the physical ageing and instability of $\mathrm{ChG}$ tapers must be considered in particular when no coating is used to protect the fibre. A poly(methyl methacrylate) coating provides two key features to subwavelength ChG tapers: high mechanical strength and chemical stability protection. Thus, the fabrication and use of uncoated ChG fibres suffer from high mechanical fragility and strong degradation. When cooling too quickly, an uncoated ChG fibre would break (fast thermal contraction). Adiabatic cooling is used to avoid taper breakage. Tapers are also mounted on a metal mount to strengthen the fibre and keep it stable. Without poly(methyl methacrylate) protection, the physical ageing of $\mathrm{ChG}$ glasses is accelerated by exposure to light and in air ${ }^{33}$. Only under controlled inert (Argon) and dry (molecular sieves) atmosphere, will uncoated tapers remain stable as reported in this work. Without these methods, quick ageing of the fibre is observed in just a few days along with degradation of the optical transmission properties.

Data availability. The data that support the plots within this paper and other findings of this study are available from the corresponding author on reasonable request.

\section{References}

1. Brown, S. Absorption spectroscopy in high finesse cavities for atmospheric studies. Chem. Rev. 103, 5219-5238 (2003).

2. Coddington, I., Swann, W. C. \& Newbury, N. R. Coherent multiheterodyne spectroscopy using stabilized optical frequency combs. Phys. Rev. Lett. 100, 013902 (2008)

3. Foltynowicz, A., Maslowski, P., Fleisher, A. J., Bjork, B. J. \& Ye, J. Cavity-enhanced optical frequency comb spectroscopy in the mid-infrared application to trace detection of hydrogen peroxide. Appl. Phys. B 110, 163-175 (2013).

4. Thorpe, M. J. Broadband cavity ringdown spectroscopy for sensitive and rapid molecular detection. Science 311, 1595-1599 (2006).

5. Thorpe, M. J. et al. Cavity-enhanced optical frequency comb spectroscopy: application to human breath analysis. Opt. Express 16, 2387-2397 (2008).

6. Faist, J. et al. Quantum cascade laser. Science 264, 553-556 (1994).

7. Yao, Y., Hoffman, A. J. \& Gmachl, C. F. Mid-infrared quantum cascade lasers. Nat. Photon. 6, 432-439 (2012).

8. Argence, B. et al. Quantum cascade laser frequency stabilization at the sub- $\mathrm{Hz}$ level. Nat. Photon. 9, 456-460 (2015).

9. Kessler, T. et al. A sub-40-mHz-linewidth laser based on a silicon single-crystal optical cavity. Nat. Photon. 6, 687-692 (2012).

10. Liang, W. et al. Ultralow noise miniature external cavity semiconductor laser. Nat. Commun. 6, 7371 (2015).

11. Siciliani de Cumis, M. et al. Microcavity-stabilized quantum cascade laser. Laser Photon. Rev. 10, 153-157 (2016).

12. Vollmer, F. \& Arnold, S. Whispering-gallery-mode biosensing: label-free detection down to single molecules. Nat. Methods 5, 591-596 (2008).

13. Orr, B. J. \& He, Y. Rapidly swept continuous-wave cavity-ringdown spectroscopy. Chem. Phys. Lett. 512, 1-20 (2011).

14. Kippenberg, T. J., Holzwarth, R. \& Diddams, S. A. Microresonator-based optical frequency combs. Science 332, 555-559 (2011).

15. Way, B., Jain, R. K. \& Hossein-Zadeh, M. High- $Q$ microresonators for mid-IR light sources and molecular sensors. Opt. Lett. 37, 4389-4391 (2012).

16. Ma, P. et al. High $Q$ factor chalcogenide ring resonators for cavity-enhanced MIR spectroscopic sensing. Opt. Express 23, 19969-19979 (2015).

17. Singh, V. et al. Mid-infrared materials and devices on a Si platform for optical sensing. Sci. Tech. Adv. Mater. 15, 014603 (2014).

18. Shankar, R., Bulu, I. \& Lončar, M. Integrated high-quality factor silicon-onsapphire ring resonators for the mid-infrared. Appl. Phys. Lett. 102, 051108 (2013).

19. Lin, H. et al. Demonstration of high-Q mid-infrared chalcogenide glass-onsilicon resonators. Opt. Lett. 38, 1470-1472 (2013).

20. Chen, Y., Lin, H., Hu, J. \& Li, M. Heterogeneously integrated silicon photonics for the mid-infrared and spectroscopic sensing. ACS Nano 8, 6955-6961 (2014).

21. Cole, G. D., Zhang, W., Martin, M. J., Ye, J. \& Aspelmeyer, M. Tenfold reduction of Brownian noise in high-reflectivity optical coatings. Nat. Photon. 7, 644-650 (2013).

22. Cole, G. D. et al. High-performance near- and mid-infrared crystalline coatings. Optica 3, 647-656 (2016)

23. Savchenkov, A. A. et al. Low threshold optical oscillations in a whispering gallery mode $\mathrm{CaF}_{2}$ resonator. Phys. Rev. Lett. 93, 243905 (2004).

24. Savchenkov, A. A., Matsko, A. B., Ilchenko, V. S. \& Maleki, L. Optical resonators with ten million finesse. Opt. Express 15, 6768-6773 (2007).

25. Grudinin, I. S., Ilchenko, V. S. \& Maleki, L. Ultrahigh optical $Q$ factors of crystalline resonators in the linear regime. Phys. Rev. A 74, 1-9 (2006).

26. Hofer, J., Schliesser, A. \& Kippenberg, T. J. Cavity optomechanics with ultrahigh- $Q$ crystalline microresonators. Phys. Rev. A 82, 031804 (2010).

27. Lin, G., Diallo, S., Henriet, R., Jacquot, M. \& Chembo, Y. K. Barium fluoride whispering-gallery-mode disk-resonator with one billion quality-factor. Opt. Lett. 39, 6009-6012 (2014).

28. Henriet, R. et al. Kerr optical frequency comb generation in strontium fluoride whispering-gallery mode resonators with billion quality factor. Opt. Lett. 40, 1567-1570 (2015).

29. Herr, T. et al. Temporal solitons in optical microresonators. Nat. Photon. 8, 145-152 (2013).

30. Liang, W. et al. High spectral purity Kerr frequency comb radio frequency photonic oscillator. Nat. Commun. 6, 7957 (2015). 
31. Grudinin, I. S., Mansour, K. \& Yu, N. Properties of fluoride microresonators for mid-IR applications. Opt. Lett. 41, 2378-2381 (2016).

32. Savchenkov, A. A. et al. Generation of Kerr combs centered at $4.5 \mu \mathrm{m}$ in crystalline microresonators pumped with quantum-cascade lasers. Opt. Lett. 40, 3468-3471 (2015).

33. Cai, M., Painter, O. \& Vahala, K. J. Observation of critical coupling in a fiber taper to a silica-microsphere whispering-gallery mode system. Phys. Rev. Lett. 85, 74-77 (2000).

34. Spillane, S., Kippenberg, T., Painter, O. \& Vahala, K. Ideality in a fiber-tapercoupled microresonator system for application to cavity quantum electrodynamics. Phys. Rev. Lett. 91, 043902 (2003).

35. Li, H. H. The infrared absorption coefficient of alkali halides. Int. J. Thermophys. 1, 97-134 (1980).

36. Hass, M. \& Bendow, B. Residual absorption in infrared materials. Appl. Opt. 16, 2882-2890 (1977)

37. Bendow, B., Lipson, H. G. \& Mitra, S. S. Multiphonon infrared absorption in highly transparent $\mathrm{MgF}_{2}$. Phys. Rev. B 20, 1747-1749 (1979).

38. Lines, M. E. Ultralow-loss glasses. Annu. Rev. Mater. Sci. 16, 113-135 (1986).

39. Gorodetsky, M. L., Savchenkov, A. A. \& Ilchenko, V. S. Ultimate Q of optical microsphere resonators. Opt. Lett. 21, 453-455 (1996).

40. Baker, C. \& Rochette, M. Highly nonlinear hybrid AsSe-PMMA microtapers Opt. Express 12, 12391-12398 (2010).

41. Ahmad, R., Baker, C. \& Rochette, M. Fabrication of Bragg gratings in subwavelength diameter $\mathrm{As}_{2} \mathrm{Se}_{3}$ chalcogenide wires. Opt. Lett. 36, 2886-2888 (2011).

42. Eggleton, B. J., Luther-Davies, B. \& Richardson, K. Chalcogenide photonics. Nat. Photon. 5, 141-148 (2011)

43. Charpentier, F. et al. Aging process of photosensitive chalcogenide films deposited by electron beam deposition. J. Alloys Compd. 509, 7330-7336 (2011).

44. Fomin, A. E., Gorodetsky, M. L., Grudinin, I. S. \& Ilchenko, V. S. Nonstationary nonlinear effects in optical microspheres. J. Opt. Soc. Am. B 22, 459-465 (2005).

45. Trebaol, S., Dumeige, Y. \& Féron, P. Ringing phenomenon in coupled cavities: application to modal coupling in whispering-gallery-mode resonators. Phys. Rev. A 81, 043828 (2010).

46. Schwarzl, T. et al. Mid-infrared high finesse microcavities and vertical-cavity lasers based on IV-VI semiconductor $/ \mathrm{BaF}_{2}$ broadband Bragg mirrors. J. Appl. Phys. 101, 093102 (2007).

47. Alligood Deprince, B., Rocher, B. E., Carroll, A. M. \& Widicus Weaver, S. L. Extending high-finesse cavity techniques to the far-infrared. Rev. Sci. Instrum. 84, 075107 (2013).

48. Yi, X., Yang, Q.-F., Yang, K. Y., Suh, M.-G. \& Vahala, K. Soliton frequency comb at microwave rates in a high-Q silica microresonator. Optica 2, 1078-1085 (2015)
49. Brasch, V. et al. Photonic chip-based optical frequency comb using soliton Cherenkov radiation. Science 351, 357-360 (2016).

50. Segelstein, D. The Complex Refractive Index of Water (MS Thesis, Univ. Missouri-Kansas City, 1981).

\section{Acknowledgements}

We acknowledge helpful discussions with Yanne Chembo. We acknowledge technical support from Arnaud Magrez, Qubig GmbH and IRflex Corporation. This work was gratefully supported by the Defense Advanced Research Program Agency (DARPA) under the PULSE programme and by grant No. 163864 from the Swiss National Science Foundation (SNSF). C.L. gratefully acknowledges support from the European Commission through Marie Skodowska-Curie Fellowships: IEF project 629649. M.L.G. acknowledges support by the Ministry of Education and Science of the Russian Federation project RFMEFI58516X0005.

\section{Author contributions}

C.L. and C.J.-G. conceived and constructed the experimental set-up, performed simulations, fabricated the samples and conducted the experiments; C.L. and C.J.-G. analysed the data with support from M.L.G.; all authors discussed the results and commented on the manuscript; C.L. and C.J.-G. prepared the manuscript; T.J.K. supervised the project.

\section{Additional information}

Competing financial interests: The authors declare no competing financial interests.

Reprints and permission information is available online at http://npg.nature.com/ reprintsandpermissions/

How to cite this article: Lecaplain, C. et al. Mid-infrared ultra-high- $Q$ resonators based on fluoride crystalline materials. Nat. Commun. 7, 13383 doi: 10.1038/ncomms13383 (2016)

Publisher's note: Springer Nature remains neutral with regard to jurisdictional claims in published maps and institutional affiliations.

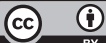

This work is licensed under a Creative Commons Attribution 4.0 International License. The images or other third party material in this article are included in the article's Creative Commons license, unless indicated otherwise in the credit line; if the material is not included under the Creative Commons license, users will need to obtain permission from the license holder to reproduce the material. To view a copy of this license, visit http://creativecommons.org/licenses/by/4.0/

(C) The Author(s) 2016 